\title{
Linx
}

Revue des linguistes de l'université Paris X Nanterre

$8 \mid 1996$

Du dire et du discours

\section{Le « sens » et l' « information » chez Harris}

\section{Danielle Leeman}

\section{OpenEdition}

\section{Journals}

Édition électronique

URL : http://journals.openedition.org/linx/1182

DOI : 10.4000/linx.1182

ISSN : 2118-9692

\section{Éditeur}

Presses universitaires de Paris Nanterre

\section{Édition imprimée}

Date de publication : 1 septembre 1996

Pagination : 209-220

ISSN : 0246-8743

\section{Référence électronique}

Danielle Leeman, «Le « sens » et l' « information » chez Harris », Linx [En ligne], 8 | 1996, mis en ligne le 17 juillet 2012, consulté le 01 mai 2019. URL : http://journals.openedition.org/linx/1182 ; DOI :

$10.4000 / \operatorname{linx} .1182$

Ce document a été généré automatiquement le 1 mai 2019.

Département de Sciences du langage, Université Paris Ouest 


\title{
Le « sens » et l' « information » chez Harris
}

\author{
Danielle Leeman
}

En hommage à Denise, cette modeste analyse d'un ouvrage du fondateur de la discipline dans laquelle elle s'est illustrée.

1 Harris fait explicitement apparaître le mot «information" dans le titre de ses deux derniers ouvrages: Language and Information et A Theory of Language and Information ${ }^{1}$. Contrairement à ce que l'on voit souvent affirmé, Harris parle du sens et s'en préoccupe, éventuellement s'y réfère dans sa théorie ou sa méthodologie grammaticale ${ }^{2}$, mais c'est la première fois, à ma connaissance, que « information » apparaît dans des titres, et il m'a semblé intéressant d'étudier en quoi l'information (en anglais : "information ») se distinguait du sens (en anglais : « meaning ») ${ }^{3}$.

\section{Harris : une syntaxe qui n'a pas de sens?}

2 Ce qui est formel, chez Harris, c'est la méthode d'identification et de description des unités linguistiques, "defined by their frequency of occurrence relative to each other, rather than by phonetic or semantic properties » (1988, VII). La théorie de la structure linguistique peut être dite formelle en ceci que les éléments sont caractérisés par l'observation de leurs distributions et des relations instituables entre ces distributions, mais il ne faut pas oublier que les résultats de cette observation sont tributaires de ce que Harris appelle "l'acceptabilité », ou la «probabilité» ou "vraisemblance de cooccurrence ", dont on ne sait pas trop sur quoi se fonde son sentiment. D'un côté, on n'a pas l'impression que l'intuition repose sur le sens lorsque l'on juge que essayer à est agrammatical si l'on entend un étranger dire J'essaie à travailler plutôt que J'essaie de travailler: c'est une erreur de construction mais l'interprétation est claire, et la même, dans les deux cas. D'un autre côté, on peut expliquer par le sens pourquoi le français dit essayer de plutôt que essayer à (et s'essayer à plutôt que s'essayer de), pourvu que l'on se 
donne une théorie sémantique des prépositions et en particulier de l'opposition entre de et $\grave{a}^{4}$. Donc on ne sait pas vraiment, lorsque l'on exerce son intuition, si l'on fait appel seulement au sentiment (inconscient) que l'on a du fonctionnement du système formel, ou seulement au sentiment (inconscient) que l'on a de la structuration sémantique - ou des deux, sans savoir comment ils s'articulent ou sont corrélés. Autrement dit, les critères qui président à l'observation des formes ne sont pas clairement définis, et Harris a parfaitement conscience du recours au sens que cela peut cacher, puisqu'il cherche, sans y parvenir, à y échapper ${ }^{5}$ et que l'un des intérêts, selon lui, de l'analyse de discours scientifiques est précisément qu'en l'occurrence "the structure be worked out with no assist from our view of its information » $(1988,40)$.

3 Ce qu'il ne faut pas oublier non plus, c'est que le sens est bien présent dans les grammaires harrisiennes - et dès le début ${ }^{6}$-, non pas comme principe présidant à l'organisation des formes (ou, du point de vue de l'analyse, à sa découverte) mais, au contraire, comme son résultat :

each entity and relation makes a fixed contribution to the meaning of its sentence;

this meaning is thus obtained directly from the words and structure of the sentence (1988, VII).

$4 \quad$ Il faut donc soigneusement décrire les distributions des mots et les relations (formelles) qui existent entre les phrases dans lesquelles ils apparaissent pour pouvoir parler du sens: l'inventaire distributionnel et syntaxique est un préalable à la saisie du sens puisque ce dernier est construit par les propriétés (formelles) des unités et de leurs combinaisons. Si je prends ainsi le premier mot de la lettre $a$ du premier dictionnaire qui me tombe sous la main, je trouve abaca. A priori, ce mot ne me dit rien (il n'a pas de sens pour moi), mais si l'on m'explique que :

L'abaca pousse aux Philipines

Le fruit de l'abaca n'est pas comestible

On arrache (ou on plante) des abacas à Manille

ces contextes me permettent de déterminer que abaca est (sans doute) un nom d'arbre. Et si l'on me dit que :

L'abaca est extrait d'une sorte de bananier

Les paillassons peuvent être en abaca

Le tapis est tissé avec des fibres d'abaca

alors je conclurai que abaca peut aussi désigner une certaine matière ${ }^{7}$. Cet accès au sens $\mathrm{du}$ mot repose sur un certain savoir linguistique, celui qui fait qu'on ne peut pas dire de n'importe quelle personne ou de n'importe quelle chose aussi bien qu'elle pousse quelque part, qu'on l'arrache ou qu'on la plante, que ses fruits sont comestibles, etc. Autrement dit, les mots ne sont pas tous "équiprobables ${ }^{8}$ dans ces contextes ( $a$ fortiori dans l'ensemble de ces contextes), et c'est précisément cette non-équiprobabilité qui constitue leur identité sémantique9.

Evidemment, il se pose le problème de la poule et de l'œuf, dit encore de l'oeuf et de la poule ; pour déduire, à partir de :

On arrache (ou on plante) des abacas

que l'abaca est un arbre ou une plante, il faut savoir ce que signifient arracher ou planter, et pour le savoir, il faut savoir ce que signifient les mots des contextes où ils sont susceptibles d'apparaître, pour la compréhension desquels... etc.! A ma connaissance, ce problème n'est pas résolu, puisque les "primitifs » eux-mêmes sont des mots qu'il faut définir ${ }^{10}$, que leur caractère même de "primitifs » peut être contesté ${ }^{11}$, et que l'on n'échappe pas à la difficulté en utilisant des symboles non linguistiques, puisque ces 
derniers doivent être définis à l'aide de mots de la langue naturelle ${ }^{12}$. Le problème est moins d'emblée crucial en syntaxe - d'où l'intérêt peut-être d'admettre qu'elle est première, le sens en étant issu, et non l'inverse - du fait que l'observation permet de décrire des structures minimales " without recourse to any externally defined elements » $(1988,15)$. En pratique, l'inventaire des distributions ou des propriétés syntaxiques repose sur une " utilisation faible du sens $»^{13}$, c'est-à-dire réduite à un jugement de similitude et de différence qui doit de toute façon trouver sa justification dans les formes ${ }^{14}$ : on peut planter des arbres ou des clous, mais on ne parlera de plant ou de plantation que pour les premiers, et si l'on peut aussi bien planter des arbres dans son jardin que planter son jardin d'arbres, on plante des clous dans ou sur le mur mais on ne parlera pas d'un mur planté de clous - sinon, peut-être, justement, par figure ${ }^{15}$. Quand bien même on ne verrait pas spontanément de différence dans le sens de planter selon qu'il s'agit d'arbres ou de clous, la langue nous oblige à en poser une puisque les propriétés du verbe (et de la phrase) diffèrent selon le complément ${ }^{16}$.

6 Une bonne partie du travail de l'équipe de Maurice Gross, loin de consister en l'élaboration de listes infinies et sans principe ${ }^{17}$, vise au contraire à établir les bases d'une " approche objectivée du sens ${ }^{18}$ en inventoriant les distributions et en les classant selon leurs propriétés transformationnelles (morphologiques et syntaxiques) afin de déterminer à quel(s) critère(s) formel(s) on peut relier le(s) sens.

\section{«Sens » et « information » : de simples variantes ?}

7 Dans la mise au point précédente, le mot « sens » a été utilisé de manière indéterminée : il peut aussi bien s'agir du rapport au référent que de la relation à l'idée qu'on s'en fait, ou du signifié entendu comme une construction propre à la langue - indépendamment de la réalité dont on parle ou telle qu'on la conçoit ${ }^{19}$-, de l'interprétation en discours ou abstraction faite de la situation ${ }^{20}$. Dans un certain nombre de cas, Harris emploie de la sorte «sens» de façon informelle - si l'on peut dire - et «information» en est le synonyme apparent :

(1) ...every step in the construction of a sentence has an established and largely unchanging contribution to the meaning of every sentence in which it occurs. There is thus an inherent relation between form and information in language (1988, VIII).

L'affirmation que chaque étape de la construction de la phrase contribue au sens de cette phrase se conclut (thus) par celle qu'il y a une relation entre la forme et l'information; de la sorte l'histoire syntaxique apparaît comme l'équivalent de la forme et le sens comme celui de l'information.

Dans d'autres cas, il n'y a pas d'équivalence portée par le texte : c'est l'un ou l'autre terme qui a été choisi, sans que l'on puisse déterminer s'ils sont ou non interchangeables ( $a$ priori, ils le sont). Ainsi, il est ci-après question d'une corrélation entre une séquence sonore et le sens, mais rien n'empêcherait de parler d'une corrélation entre une séquence sonore et l'information :

(2) The one of element that is precisely established is the set of phonemes (...). But as the words, if they are thought of as correlations of sound sequences with meanings, we are left with many problems $(1988,2)$.

10 Rien ne s'oppose non plus apparemment à parler de sens là où «information » a été choisi : 
(3) The structure of each science language is found to conform to the information in that science rather than to the grammar of the whole language (...).

(4) The third lecture is a discussion of information, in particular as it appears in language. This is not an added or independant issue, but an intrinsic concomitant of the structural analysis (1988, VIII).

11 Dans la citation (3), « information » semble référer aux savoirs construits par la science, donc à des représentations spécifiques, et s'oppose à la « grammaire » de la langue : on pourrait en déduire que l'information est en quelque sorte technique, renvoyant à une connaissance élaborée selon des règles propres et qui ne coïncide pas avec ce que véhicule la langue (ordinaire), qui serait le sens ${ }^{21}$. De même, c'est «information " (à travers l'adjectif correspondant) qui est choisi pour qualifier le métalangage d'un champ donné : « a language of broader informational capacity than the given field » $(1988$, p. 2$)$, mais semantic (et non informational) lorsqu'il s'agit des propriétés des mots et des phrases dans la langue ordinaire : « words and sentences exist, not merely by cultural convention or by some semantic properties, but by restrictions of combination (...) » $(1988$, p. 6).

Mais la citation (4) contredit cette interprétation, puisque l'“information" y est cette fois associée à la langue (ordinaire) - bien qu'il soit sous-entendu qu'il y en ait d'autres types, qui n'apparaissent pas dans la langue ${ }^{22}$. Et c'est semantic - et non informational - qui apparait dans : «These are the major specific properties of the base of derived sentences (...). They give language its form and its semantic capabilities » (ibid., p. 30).

13 Lorsque l'on regarde à propos de quoi il est question d' « information » et de " sens », on peut encore penser à une autre répartition : l'information serait le fait du système, langue ou sous-langage (scientifique) - citations (1), (3), (4) ${ }^{23}$ - mais le sens concernerait les unités : phrase - citation (1) - ou mot - citation (2). De même, on observe :

(5) ...a behavioral test that does not involve the specific meaning of words (p. 4).

The phoneme sequence relation that makes words (...) does not in general ties up with the other properties of words -nor with their meanings (p. 6).

...the word sequence relations are explicit and are of decisive importance for the structure and meaning of sentences (p. 6).

14 (Il y a d'autres exemples pp. 15, 29, 30, 32, 35, 50, etc.). Le sens peut aussi être celui des contraintes de combinaison, d'une transformation ou d'une catégorie grammaticale :

(6) ...there are three types of constraint of word combination that make sentences, and each one carries a type of meaning, so that the meaning of a sentence is determined directly from the words and the constraints. (...): a probability inequality that allows for words meaning (...) (p. 10).

(7) The partial-order relation has a meaning: as will be seen later, each operator is being said about its argument, so that the meaning of the partial order is roughly predication (p. 13).

(8) ...the linear order among coarguments is an additional constraint (with its own "grammatical» meaning), and the linearization of the partial order (which contributes no meaning) is an additional constraint (...) (p. 26).

(9) Such grammatical paradigms give prominence to particular grammatical meanings such as tense, plurality, and person (p. 30).

Mais contradictoirement c'est «meaning » et non « information » qui est choisi en cooccurrence avec « all language » dans :

(10) ... almost all further classes and operations in language, and almost all language meanings, are formulated on the constructions resulting from this relation (the dependance-on-dependance relation) (p. 15). 
Inversement, c'est «information » et non «meaning» qui apparaît avec «word combination » dans :

(11) The more stringent boundaries and tighter interrelations in the subject matter are reflected in sharper correspondance between word combination and information (p. 40).

avec « sentences » dans :

(12) ...the gross formulas show the frame-work of the science information, while the detailed ones represent the information carried by individual sentences (p. 52). et le sens est dévolu aux mots et l'information aux phrases dans :

(13) ...we can ask how words carry meaning and how sentences carry information (p. 57).

Ainsi la répartition n'est pas: le «sens" pour les unités ou les opérations vs l'“information" pour la langue vue dans son entier. Ce qui fait penser que les deux termes ne sont toutefois pas interchangeables, c'est que ${ }^{24}$ :

(14) Even the meanings of words (...) are in part determined from their combinations rather than purely from their identity (p. 15).

où l'on voit que le sens (des mots, en l'occurrence) comporte deux aspects : il est en partie déterminé par les propriétés distributionnelles et syntaxiques, il ne provient pas seulement de l'identité des mots ; c'est donc qu'il y a deux faces dans le « sens ", l'une qui serait construite par la langue, et l'autre qui serait intrinsèque au mot, en quelque sorte l'image qui surgit mentalement si l'on rencontre le mot isolément ${ }^{25}$ :

(15) Whereas the first constraint (the partial order constraint) creates sentence structure, the second (the likehood constraint) specifies word meanings. It does not necessarily create meaning, since many words with their meanings must have been in use singly before being used in sentences, but it specifies meaning to any detail desired, and it enables a word to extend its meaning, and to have different meanings in different operator-argument environments (p. 16 - c'est moi qui souligne en gras).

L'« information » serait donc tout ce qui concourt, par un témoignage formel (comme illustré à propos de planter), à l'interprétation du mot ou de la phrase : le mot en tant qu'il est défini par ses propriétés distributionnelles, morphologiques, syntaxiques, et la phrase en tant qu'elle est constituée de mots et entre dans tel et non tel autre ensemble de transformations - en somme le sens en tant qu'il est construit explicitement par la langue ${ }^{26}$.

Mais " meaning » (le "sens ») est ambigu, étant susceptible de désigner soit le concept évoqué par le mot isolé, soit l'ensemble formé par le concept et ce que construisent les propriétés linguistiques ; rappelons la citation (1) :

...the construction of a sentence has (a) contribution to the meaning of every sentence

et ajoutons-y celle-ci :

We have seen the constraints on word combination: the partial order of word dependence that created sentence structure, the likehood inequalities that fit word meanings (...) (p. 27).

S'il n'est pas question de « sens » à propos des textes scientifiques (citation (3), c'est parce qu'il se confond entièrement avec l'«information", en ceci que les concepts sont exhaustivement décrits (et donc appréhendables) par les propriétés linguistiques des mots et des phrases où ils apparaissent ${ }^{27}$ : il n'y a pas comme dans la langue ordinaire de « reste » hors du champ de l'analyse grammaticale (cf. le almost de la citation (10)) ni d'interprétation qui viendrait de l'extérieur du texte, comme lorsque l'on parle de 
«prédication » pour la relation entre l'opérateur et l'argument (citation (7)) : disant que dormir est le prédicat de bébé dans Le bébé dort, j'étiquette la connexion par un terme qui n'est pas dans la phrase ${ }^{28}$.

Ainsi, « sens » et « information » ne sont pas de simples variantes, mais si le second a un statut clair, le premier est ambigu.

\section{Distinction et rapports mutuels entre « sens " et « information»}

La citation (13) associe explicitement le « sens » au mot et l'« information » à la phrase : le sens concerne le mot en ceci que ses propriétés formelles ne disent pas ce qu'il signifie, ce à quoi il réfère ${ }^{29}$; on sait que planter a plusieurs interprétations (puisqu'il entre dans divers ensembles distributionnels, morphologiques, syntaxiques) mais on ne sait pas lesquelles ${ }^{30}$. Réciproquement, le sens seul ne permet pas de prédire le comportement linguistique du mot (boire est traditionnellement défini comme « avaler un liquide » mais on ne dit pas boire son potage quoique le potage soit un liquide ${ }^{31}$ ) - il ne suffit même pas à l'identifier ${ }^{32}$, du fait que l'on ne dispose pas d'un système sémantique explicite défini indépendamment de la langue (hormis dans quelques cas restreints, comme celui des nombres) à partir duquel on pourrait établir des relations entre ses composants et ceux de la langue ${ }^{33}$ :

(meanings) can give a property to words that are already identified, but they don't identify words (p. 60).

L'information concerne la phrase, puisque c'est l'inscription du mot dans un contexte (susceptible de telles et non telles transformations) qui fait surgir son interprétation (ce qu'il signifie) : si la grammaire ne peut décrire le sens, elle peut décrire l'information, c'est-à-dire ce qui, dans le sens, est mis en forme par la langue ${ }^{34}$.

L'information se trouve essentiellement représentée par deux contraintes fondamentales qui déterminent la phrase : d'une part la concaténation d'opérateurs à leurs arguments et d'autre part le choix des mots dans chacune des catégories ${ }^{35}$. Ces contraintes sont régulières, stables (elles se retrouvent quelle que soit la construction: L'enfant dort, L'enfant dort-il ?, C'est l'enfant qui dort, etc.) et donc :

The contributions these constraints make to the meaning of their sentence is fixed

for all the occurrence of these contraints (p. 59).

(Il s'agit seulement de « contributions » parce que, comme on l'a vu, les contraintes sont solidaires d'une interprétation dont elles sont la marque, mais elles n'en donnent pas la définition : elles ne disent pas ce que signifie la phrase ${ }^{36}$.)

Ainsi, le mot par lui-même ne peut relever de l'“information", puisque celle-ci consiste en un ensemble de propriétés qui ne sont saisissables qu'à travers la phrase, la combinaison de mots (citations (2), (5), (13), etc.). Si le mot ne peut avoir que du sens, en revanche la phrase peut être appréhendée de deux points de vue: elle réfère (à un événement, un état, une relation) et donc a un sens, mais sa composition même et son rapport à d'autres ont une incidence sur le choix de l'interprétation et donc relèvent de l'information (d'où à la fois les citations (1), (5) et (11), (12), (13) par exemple). Les opérations ou les catégories grammaticales relèvent évidemment de l'information mais ont aussi un sens (les morphèmes «tense » ou "plurality » réfèrent). La langue elle-même est un système d'information (c'est-à-dire de codage formel des interprétations) mais dont les unités (les 
mots, les phrases) ont un sens, d'où la possibilité de parler des "language meanings » (citation (10)).

Bien qu'il arrive à Harris de l'écrire ${ }^{37}$, on ne peut pas exactement dire que la langue "véhicule» (de) l'information (comme on dit que le mot a un sens ou que la phrase possède une interprétation): telle qu'on l'a définie, l'information se confond avec la structure (formelle) linguistique ${ }^{38}$; elle n'en est pas l'objet, ou la conséquence, la langue n'est pas une forme qui la contiendrait ou la secréterait ${ }^{39}$ : la langue n'est telle qu'en tant que forme-contenu, qu'en tant que «structure de l'information » où "structure » et « information » seraient co-référents (comme dans la région du Nord).

La relation avec les référents, c'est-à-dire de la langue avec le monde tel que nous le percevons, est peut-être plus étroite qu'il n'a été affirmé jusqu'à présent: du moins les sous-langages scientifiques témoignent de discours étroitement solidaires des événements ou des objets dont ils parlent, si bien que l'information s'y confond avec le sens. Cet exemple suggère

that the power of the sentences of language to refer to the events of the world, and more particularly the power of science language sentences to refer to the events of a science, involves some kind of similarity in the way that what is common to both the underlying departures from randomness- is structured in a set of events, on one hand, and in a set of sentences, on the other (p. 85).

\section{NOTES}

1. Harris, Z. (1988) Language and Information, New York, Columbia University Press ;- Harris, Z. (1991) A Theory of Language and Information, Oxford, Clarendon Press.

2. Par exemple, à propos de gamma globulin dans un texte d'immunologie : "what is meant (...) here is just antibody" et de même : "immunize was used synonymously with inject, and normal was to mean not injected" (1988, p. 43).

3. La place étant mesurée, je me bornerai à l'étude des deux termes dans le premier des ouvrages cités, quoiqu'ils apparaissent ailleurs antérieurement.

4. Cadiot, P. (1993) "De et deux de ses concurrents : avec et $\grave{a}$ ", Langages 110, Paris, Larousse.

5. "Le voisinage contextuel pourrait être utilisé comme critère de paraphrase, ce qui éviterait de rechercher s'il y a ou non variation de sens. Toutefois, cela n'a pas encore été étudié" (Harris,

Z. (1968 trad. 1971) Structures mathématiques du langage, Paris, Dunod).

6. On lit dès la page 7 de Structural Linguistics que life et rife "differ in distribution (and hence in "meaning")" (1951, Chicago and London, Phoenix Books). Cf. aussi dans ce même ouvrage entre autres les pages 186-195: "The criterion of meaning", et les articles traduits en français : (1952 trad. 1969) "Analyse du discours", Langages 13, Paris, Larousse, et (1954 trad. 1970) "La structure distributionnelle", Langages 20, Paris, Larousse.

7. Même pour des mots connus, ordinaires, le sens n'apparaît qu'en contexte, ce que montrent les gloses des dictionnaires y compris quand ceux-ci ne se préoccupent ni de la morphologie ni de la syntaxe pour définir le sens du mot. Par exemple, on opposera boire un liquide et boire sans complément.

8. Cf. Harris (1954 trad. 1970). 
9. On peut à l'inverse considérer que c'est le sens qui est responsable des propriétés distributionnelles ou syntaxiques, ou que les deux sont indépendants, ou encore qu'ils sont interdépendants (il reste alors à déterminer leur symbiose).

10. Cf. Wierzbicka, A. (1993) "La quête des primitifs sémantiques", Langue française 98, Paris, Larousse.

11. Cf. Desclès, J.-P. (1987) "Réseaux sémantiques" et Rastier, F. (1987) "Représentation du contexte lexical", Langages 87, Paris, Larousse.

12. Cf. Rey-Debove, J. (1978) Le métalangage, Paris, Le Robert. Harris évite cette régression infinie (1988, pp. 35 sq.).

13. Termes de Henry Hiz cités par Leeman, D. (1973) "La paraphrase dans l'oeuvre de Hiz", Langages 29, Paris, Larousse.

14. Ce principe généralement admis dans la communauté des linguistes est appelé par JeanClaude Milner : "principe de naturalité" (Milner, J.-C. (1989), Introduction à une science du langage, Paris, Le Seuil).

15. Sur le verbe planter, cf. Boons, J.-P. (1974) "Acceptabilité, interprétation et connaissance du monde à propos du verbe planter", Actes du colloque franco-allemand de Grammaire Transformationnelle, Linguistische Arbeiten 14, Tübingen, Max Niemeyer Verlag.

16. De même, on peut ne pas voir d'emblée l'ambiguïté dans Le patron a augmenté ses ouvriers, qui n'en est pas moins reliable à deux ensembles distincts : Le patron a augmenté le nombre des ouvriers/ les effectifs (les ouvriers augmentent/sont en augmentation) et Le patron a augmenté le salaire des ouvriers (Les ouvriers ont été augmentés/ont obtenu une augmentation: Le patron a accordé une augmentation aux ouvriers). Cet exemple est de Giry-Schneider, J. (1994) "Sélection et sémantique, problèmes et modèles (présentation)", Langages 115, Paris, Larousse (p. 10).

17. "Toute méthode distributionnelle aboutit rapidement à des constats infinis et sans principe. Ce que montrent à l'envi les dictionnaires et les recensements de l'école de Maurice Gross" (Marandin, J.-M. (1992) "L’individualité lexicale, III. Il y a de la synonymie", Cahiers de lexicologie 61, Paris, Didier Erudition, p. 54).

18. Gross, M. (1986) Grammaire transformationnelle du français : l'adverbe, Paris, ASSTRIL (p. 32).

19. Sur les difficultés à définir le sens, cf. Normand, C. (1990) La quadrature du sens, Paris, PUF.

20. Sur "sens" et "signification", cf. Ducrot, O. (1980) "Texte et énonciation", dans Les mots du discours, Paris, éditions de Minuit.

21. Ainsi a-t-on longtemps défini la chauve-souris comme un oiseau et la baleine comme un poisson, alors qu'elles étaient considérées comme des mammifères par les savants (Lagane, R. (1969) "Problèmes de définition. Le sujet", Langue française 1, Paris, Larousse).

22. On peut penser aux gestes, aux mimiques, à la couleur du ciel (qui annonce un orage), aux gargouillis d'un estomac (qui renseignent sur la faim de son possesseur), etc.

23. D'autres exemples: "And, as will be seen, the base suffices for all the information carried by the language" (p.28);- "...all the information expressed in the language" (p. 29) ; -"Aside from the purely structural caracteristics of these sublanguages, there is an important property in respect to information" (p. 39) ; "...the original information as given in the whole language" (p. 41). -"We obtained a gross framework for the information in the field" (p. 45); etc.

24. Cf. aussi : "language is clearly a carrier of meaning and information" (1988, p. 57).

25. On associe toujours un concept à une suite sonore ou graphique jusque là inconnue; ainsi, une petite enquête menée auprès d'étudiants montre que, pour ceux qui n'ont pas lu Pagnol, le mot bartavelle est susceptible d'évoquer pour les uns une danse, pour d'autres une fleur, ou une embarcation, etc. - où l'on voit sans doute l'écho des "associativités" dont parle Saussure (Saussure de, F. (1916), Cours de linguistique générale, Paris, Payot, p. 175).

26. Comme dans la théorie de l'information, "information" désigne donc le sens en tant qu'il est codé dans le système. La relation entre la probabilité d'occurrence et le sens apporté (inversement proportionnels) lui est d'ailleurs aussi empruntée explicitement : "The words that 
have highest likehood, i.e. are expectable, in a given environment contribute little or no information when they enter there, in the information-theoretic sense" (p. 20).

27. Harris (1988) présente dans le chapitre II le résultat d'analyses de différents corpus scientifiques (dans le domaine de l'immunologie) publiés de 1935 à 1966, rassemblées dans Harris, Z. et alii (1987) The Form of Information in Science: Analysis of an Immunology Sub-Language, Dordreich, Reidel. Cf. aussi à ce propos : Daladier, A. (1990) (dir.) Les grammaires de Harris et leurs questions, Langages 99, Paris, Larousse.

28. On fait la même chose en parlant de "tense", "plurality", "person", etc. dans le domaine des catégories grammaticales.

29. To refer to, to indicate, to mean sont équivalents (Harris, 1988, p. 66). Un mot réfère aux "properties common to its possible set of referents" plutôt qu'aux "objects or events or states or relations" eux-mêmes puisqu'un mot peut correspondre à un référent d'extension nulle sans pour autant avoir un sens nul: "Thus unicorn and centaur may be indistinguishable in the extension of their referents, since no object exist to which either refers" (p. 64).

30. Du moins la grammaire ne nous le dit pas mais nous en avons une idée si nous connaissons la langue (ou si nous consultons un dictionnaire).

31. Le Petit Robert (1983) définit ainsi potage: "bouillon dans lequel on a fait cuire des aliments solides" et bouillon : "liquide dans lequel certaines substances ont bouilli."

32. On peut penser que dans le cas des homonymes, c'est le sens qui permet d'identifier les mots ; mais, outre que le jugement porte sur des contenus déjà élaborés formellement (une séquence phonétique ou graphique), la décision qu'il y a homonymie est souvent difficile à prendre et contestable. Cf. l'analyse par Jacqueline Picoche du choix de quelques dictionnaires à ce sujet: Picoche, J. (1995), "Combien y a-t-il de coeur(s) en français ?", Langue française 105, Paris, Larousse. 33. Dans le cadre harrisien, les unités (phonèmes, morphèmes, mots) sont identifiées par un calcul de probabilité d'occurrence indépendant du sens (cf. par exemple Harris (1951) et (1968). On n'a pas l'équivalent dans le domaine sémantique, c'est-à-dire une méthode d'identification des unités indépendamment de leur mise en forme linguistique.

34. Mais en même temps le sens (en tant qu'il échappe à la grammaire: appelons-le "interprétation") est lié à l'information puisque les propriétés formelles diffèrent selon l'interprétation, et l'information est liée au sens (à l'interprétation) puisque les phrases sont faites avec les mots, et que leurs propriétés diffèrent selon les mots qui les constituent.

35. L'enfant dort est une structure de phrase, et non * l'enfant l'enfant ni * dort dort; dans cette structure, l'enfant (et autres) a un degré de probabilité d'occurrence plus grand que l'arbre, la terre , etc. (mais ces derniers ne sont pas exclus grammaticalement comme le sont dort, la, vite, etc. devant dort).

36. D'autres contraintes existent mais sans incidence substantielle sur l'information (c'est-à-dire sur la relation entre l'opérateur et l'argument): la variation morphologique (obéissance est l'opérateur de enfants dans l'obéissance des enfants comme obéir l'est dans Les enfants obéissent), la topicalisation, qui est "the speaker's indication of what he is talking about" (p. 58) - dans Ils obéissent, les enfants, le rapport entre l'opérateur et l'argument est le même que dans Les enfants obéissent; etc.

37. Par exemple p. 76 : "It has been seen that the information carried by the language (...)".

38. Ce dont semble témoigner la coordination p. 84 : "the structure of language and information" ( $v s$ the structure of language and of information ou the structure of language and the structure of information).

39. Harris parle de l'information "as some kind of concomitant of language" (p. 81) -cf. aussi la citation (4). 


\section{RÉSUMÉS}

Zellig S. Harris passe, aux yeux de certains, pour un linguiste qui se serait obstiné, quelque peu stupidement, à ne pas parler du sens. Mais si son premier ouvrage, en 1951, est une tentative de formalisation des procédures de la linguistique structurale d'identification et de classement des unités primitives du système (phonèmes et morphèmes), la syntaxe est dès cette époque conçue comme l'organisation du sens par la langue (et donc sa description le moyen pour le linguiste d'appréhender les phénomènes d'homonymie, de polysémie et de synonymie). Nous analyserons ici la distinction opérée par Harris en 1988 entre le sens et l'information - reprise et développée dans son dernier ouvrage, en 1991.

Some people hold Zellig S. Harris to be a linguist who, rather stupidly, persisted in not speaking about meaning. But, though his first work, in 1951, was an attempt to formalize the procedures of the structural linguistics of identification and classification of the system's primitive units (phonemes and morphemes), even at that time syntax was conceived as the organisation of meaning by language (and so for the linguist, its description was the means to embrace the phenomena of homonymy, polysemy and synonymy). Here we analyze the distinction, made by Harris in 1988, between meaning and information, which was picked up again and developed in his last work in 1991.

\section{AUTEUR}

\section{DANIELLE LEEMAN}

